\title{
Numerical study of self-loosening of a bolted assembly under transversal load
}

\author{
Olfa Ksentini ${ }^{1,2, a}$, Bertrand Combes $^{2}$, Mohamed Slim Abbes ${ }^{1}$, Alain Daidié2 \\ AND MOHAMEd HadDaR ${ }^{1}$ \\ 1 Dynamics of Mechanical Systems Research Unit, National School of Engineers of Sfax, BP 1173-3038, Sfax, Tunisia \\ 2 Clement Ader Institute, University of Toulouse, National Institute of Applied Sciences of Toulouse, 31077 Toulouse, France
}

Received 28 May 2015, Accepted 23 November 2015

\begin{abstract}
Self-loosening of bolts is a problem encountered in many industrial fields, and especially in aviation. The purpose of this paper is to study the self-loosening phenomenon numerically through an assembly consisting of two plates assembled by a bolt and subjected to a transversal dynamic load. First, a 3D finite element model is developed, with contact elements and all geometrical details, including the real thread geometry with its helix angle. This leads to many degrees of freedom and long calculation time. Then a 1D simulation is made, based on a simplified model with only 4 degrees of freedom and few contact conditions. Calculation is nearly instantaneous. Both models describe the succession of sticking and slipping on contact surfaces and predict loosening of the assembly. They provide close results. The next step will be to compare their behaviour with an experimental approach, in order to validate and fit the models.
\end{abstract}

Key words: Self-loosening / bolted joint / dynamic behaviour / finite element model / 1D simulation

\section{Introduction}

Bolted connections are frequently employed in mechanical structures for their easy use and maintenance. However, the problems resulting from vibrations, such as fatigue and loosening, still interest many researchers. The screw connection consists of an irreversible thread, so loosening can occur only under the influence of an external load (torque or force) which overcomes the friction forces $[1,2]$. This problem is not always well controlled for various reasons, such as faulty design, unexpected vibrations or poorly evaluated friction. Several techniques can be used to avoid self-loosening. Many experiments have been conducted to demonstrate the loosening phenomenon, and theoretical studies have been based on either analytical or numerical approaches. Results obtained in these studies showed that self-loosening can vary depending on the load direction relative to the bolt axis.

Some studies were devoted to joint loosening under axial loading. Hess $[3,4]$ applied a compressive load to a bolt through a cam. He explored the influence of preload, thread pitch and external load on the loosening. In later studies, Aziz investigated the influence of vibration on the assembly [5]. He used a shaker to apply a load acting in

${ }^{a}$ Corresponding author: olfa.ing@gmail.com the axial direction on an inertial mass. This experiment led to separation of the nut from its support but without unscrewing. All results obtained from these studies showed that it was impossible to loosen a bolt assembly using an axial load when the assembly was properly sized.

Other studies have shown that loosening generally occurs when a fastener is subjected to a transverse load. It results from slipping that occurs between the bearing surfaces of the screw, the nut and the assembled parts. Junker [6] studied the loosening of an assembly under transverse load with an eccentric cam that forced relative slipping of bolted parts. Rolling elements were interposed at the interface between parts in order to minimize friction. Junker's experimental setup has been used in many other studies as it hastens unscrewing by facilitating relative displacements. However, it does not match most industrial devices. Bhattacharya [7] developed a similar apparatus to test different types of nuts, washers and other devices in order to find the most adequate ones to counteract the loosening effect in bolted joints.

Alongside these experimental works, the literature contains many numerical studies based on finite element models. To depict the loosening phenomenon adequately, a detailed three-dimensional finite element model taking the helix geometry of thread into account is an obvious choice. However, due to the large number of elements 
associated with this model, the computation is very timeconsuming. Some recent research has dealt with the modelling of the loosening of bolted assemblies. Jiang [8] studied a curvic coupling subjected to transverse loading, while Zhang [9] addressed an assembly under several conditions of preload and externally imposed displacement. They found that loosening resulted from micro slipping under the screw and the nut thread. Avoiding selfloosening is an important step. Dinger [10] developed a three-dimensional finite element model. His study demonstrated methods for preventing the loosening of a bolted joint assembly and he compared his results with experiments conducted on a modified Junker machine. Analytical approaches were developed by Nassar [11, 12], who developed equations to study the loosening of a bolt assembly. He developed a mathematical model to show the influence of the thread pitch and the preload on the bolt self-loosening. He also validated his results by an experiment based on the modified Junker machine. Yang [13] developed a numerical model based on Nassar's studies to search for a way to avoid the loosening of a bolted joint assembly subjected to transverse loading. The effects of thread pitch and friction coefficient were investigated. He recommended using a fine thread to reduce loosening of bolted joints and he proposed several means of increasing the friction coefficient under the nut or the screw.

In this paper, a numerical study is conducted to investigate the self-loosening of an assembly consisting of two plates assembled by a bolt, subjected to a transverse displacement. First, a 3D finite element model is set up, with all the details of the thread. Local application of the Coulomb friction law brings out micro slip and enables unscrewing of the nut and loosening of the assembly to be simulated. Then a $1 \mathrm{D}$ simulation is conducted on a simplified model, using few physical variables, where the threaded connection is replaced by a simplified description, and the Coulomb friction law is applied over the entire contact surfaces. Numerical integration of the equations of motion is conducted by a Runge Kutta method to reveal the unscrewing and loosening, which are then confronted with the previous results.

\section{Model of assembly}

\subsection{Geometrical model}

The assembly is presented in Figure 1 and consists of two steel plates, assembled by a bolt. The upper plate is clamped at one end, whereas the lower plate receives a reciprocating motion in the $x$ direction, orthogonal to the axis $z$ of the screw, at the other end.

All the dimensions are given in Figure 2. Each plate is $64 \mathrm{~mm}$ wide, $134 \mathrm{~mm}$ long and $11 \mathrm{~mm}$ thick. The screw diameter is $6 \mathrm{~mm}$ and its grade is 8.8 .

\subsection{Mechanism of unscrewing}

The movement imposed at one end of the assembly causes slipping of the lower plate against the upper one, and thus bending of the screw shank as in Figure 3. So shearing forces $f_{t}$ appear on the bearing faces of the screw head and the nut and they can cause slipping of these surfaces if bending is sufficient. The bearing flank of the thread also supports shearing forces, and it, too, can slip.

On the other hand, due to the preload acting on the thread through its helix angle, both the screw and the nut receive a torque $t$ from the thread along their common axis, acting in the loosening direction. When there is no relative displacement between the assembled plates, this torque is not sufficient to cause sliding, and unscrewing cannot occur.

However, when sliding appears both on the bearing face of the screw head and on the thread, this torque deflects the sliding, causing a slight rotation of the screw and thus initiating the loosening. Similarly, when sliding appears both on the bearing face of the nut and on the thread, the nut unscrews and the assembly loosens. This phenomenon is described in reference [2] and will be discussed in greater detail in Section 4.2.

\section{Simulation of self-loosening by a finite element method}

\subsection{Meshing and interactions}

The model was encoded with Abaqus ${ }^{\circledR}$ 6.13-5 [14] using hexahedral eight-node elements and several interaction types. The number of elements was minimized as far as possible by the use of a so-called "tie constraint". This constraint is useful for refining the mesh, in particular for 3D problems. It allows the two parts to be attached and have the same motion, even if they do not have the same meshing. It thus authorizes strong transitions in mesh density. It was used to attach the screw thread to the screw shank and, in the same way, to attach the nut thread to the nut body. So a finer mesh was used only in the threads, which contained small shape details, while the other parts had a coarser mesh, thus limiting the number of elements. The whole model contained 66208 elements, 52000 of which belonged to the threads. The meshing of the thread area and the tie constraints are shown in Figure 4.

A contact constraint with Coulomb friction was applied to the surfaces likely to slip: between the upper plate and the screw head, between the two plates, between the lower plate and the nut, and between the flanks in contact of the threads of the nut and the screw, as shown in Figure 5 .

Unscrewing can occur by rotation of the screw, or the nut, or both, depending on the geometry of contacts and the effective friction coefficient at each place. In order to facilitate observation and analysis of behaviour, it was necessary to restrict the rotating movement in one part. So the friction coefficient was set to 0.1 everywhere except under the screw head, where it was set to 0.11 . Thus sliding occurred under the nut rather than under the screw head, and the screw head could never slide on the upper plate. 


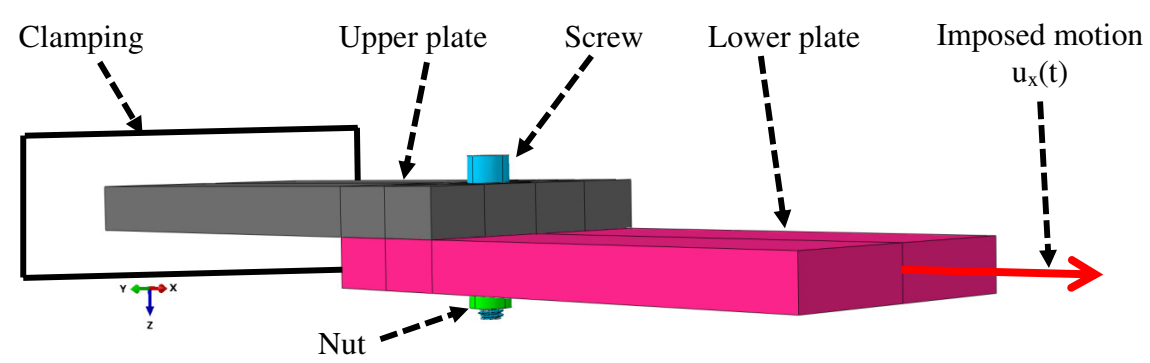

Fig. 1. Geometrical model.
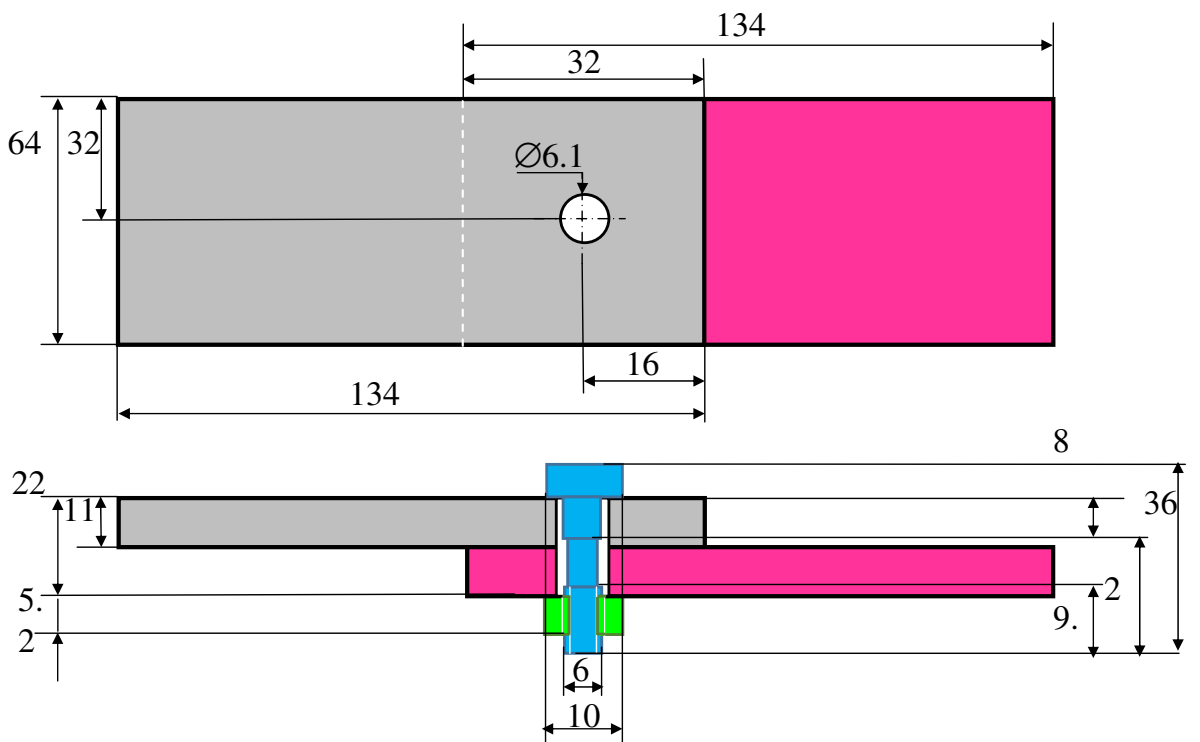

Fig. 2. Dimensions of model.

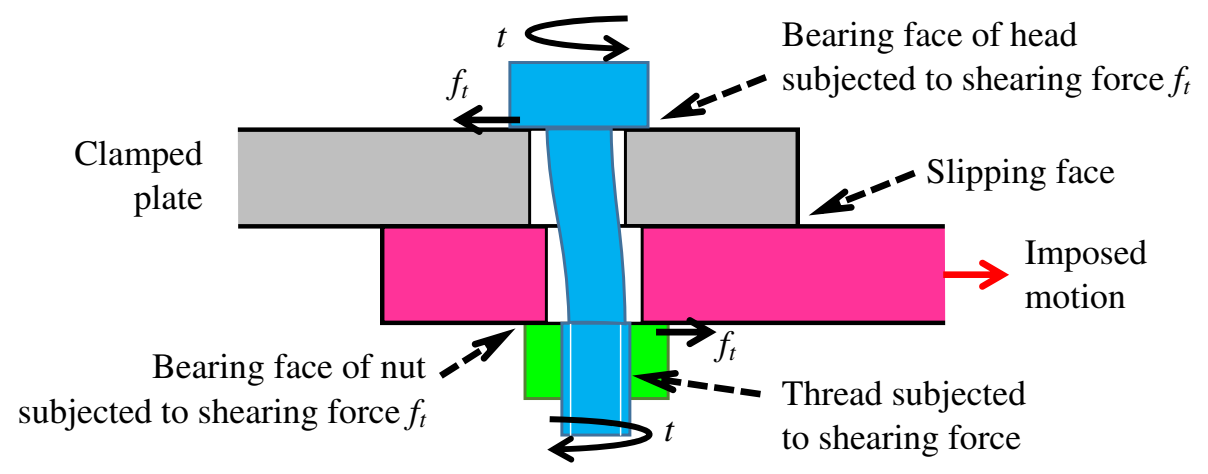

Fig. 3. Assembly distorted by effect of imposed motion.

\subsection{Calculation steps}

Three steps were performed for the dynamic analysis. In the first one, interactions and preload of the bolt were created. The end of the upper plate was fixed while the nut was prevented from rotating, and an angular rotation of $0.35 \mathrm{rad}$ was applied to the screw head. This set up an axial load in the bolt shaft equal to $8600 \mathrm{~N}$, corresponding to $68 \%$ of the screw yield stress $\left(R p_{0.2}\right)$, and a torsional moment. Then a stabilization phase took place, in which the nut was released while the screw was maintained in its last position. Finally, a sinusoidal displace- ment was applied to the end of the lower plate, according to Equation (1).

$$
u_{x}(t)=0.5 \times\left(\cos (2 \pi \times 180 \times t)^{-1}\right)
$$

A prior modal calculation was conducted and showed that the first natural mode of the structure was about $162 \mathrm{~Hz}$. So a movement with a frequency of $180 \mathrm{~Hz}$, pretty far from the first eigenfrequency, was imposed. A peak amplitude of $0.5 \mathrm{~mm}$ was chosen so that slipping occurred under the nut at each halfwave. The diagram in Figure 6 shows these 3 calculation steps. 


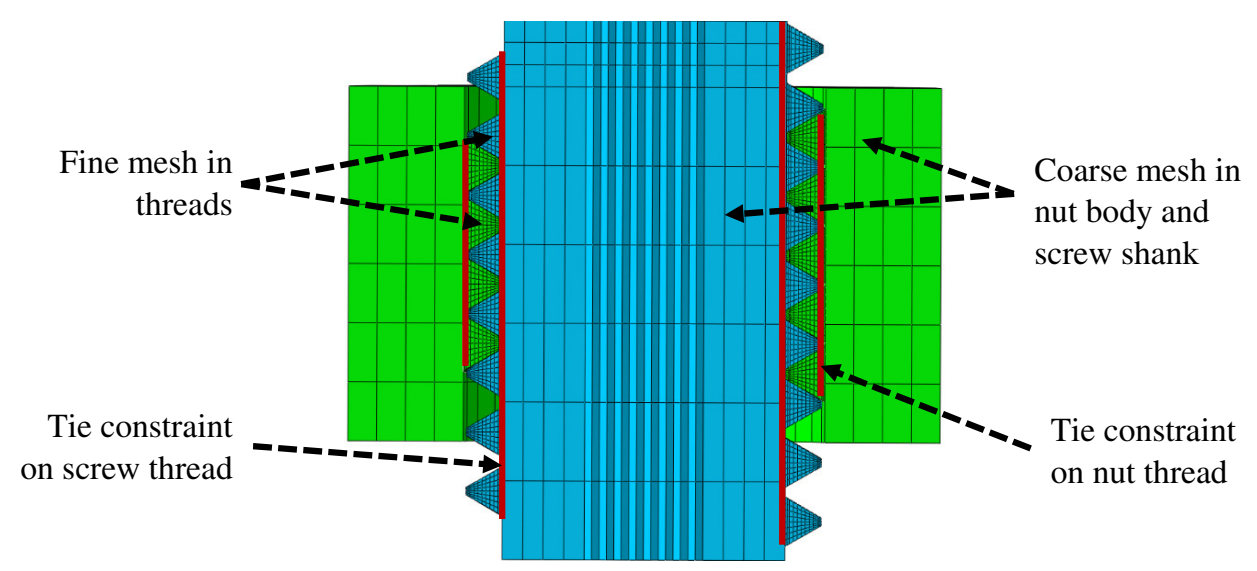

Fig. 4. Meshing of the thread area and tie constraints.

\section{Contact of screw head}

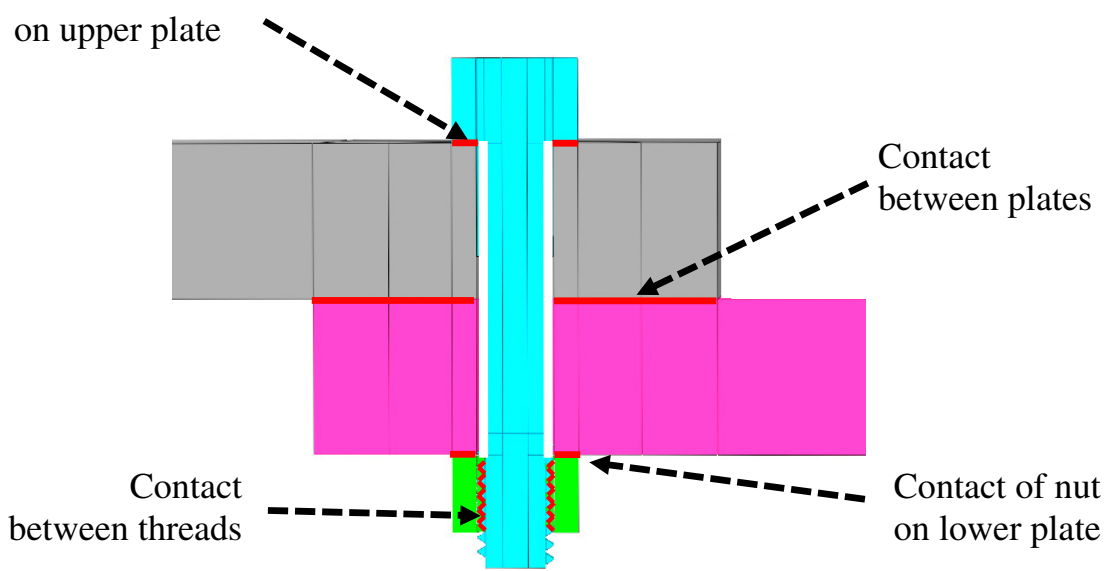

Fig. 5. Contact areas.

Step 1: Preload from $t=0$ to $t=0.008 \mathrm{~s}$

- Nut rotation is prevented

- Angular displacement $0.35 \mathrm{rad}$ in $\mathrm{z}$ direction is applied on the screw

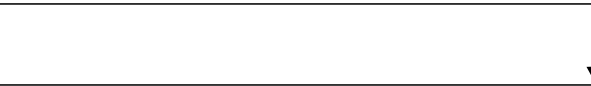

Step 2: Stabilization from $t=0.008$ to $t=0.01 \mathrm{~s}$

- The screw is maintained at its position

- The nut is released

Step 3: Vibrations from $t=0.01$ to $t=0.0322 \mathrm{~s}$

- A sinusoidal movement is applied to the lower plate:

$u_{x}(\mathrm{t})=0.5 \cdot(\cos (2 \pi \cdot 180 \cdot t)-1)$

Fig. 6. Calculation steps. 


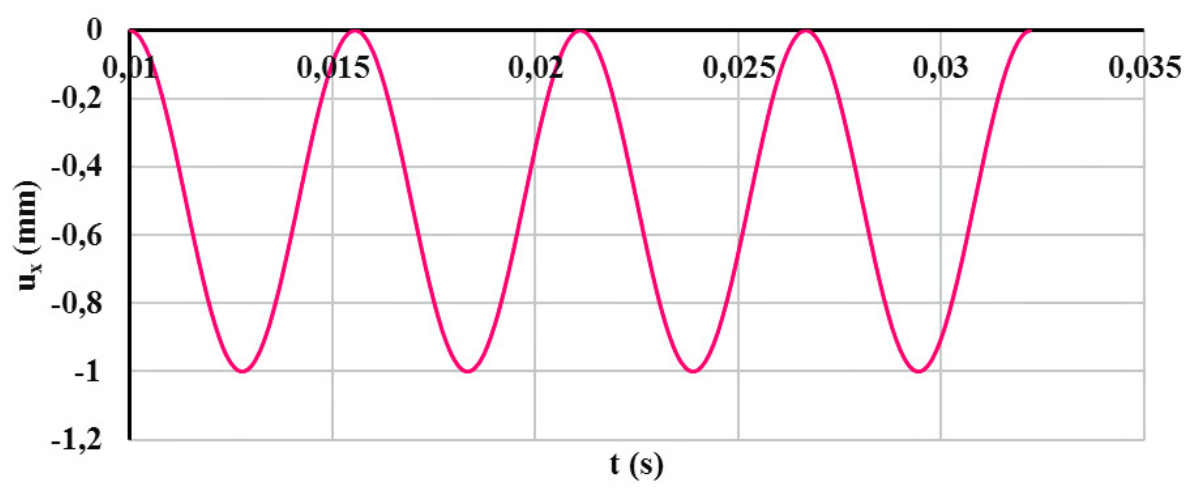

Fig. 7. Displacement $u_{x}$ at the end of the lower plate.

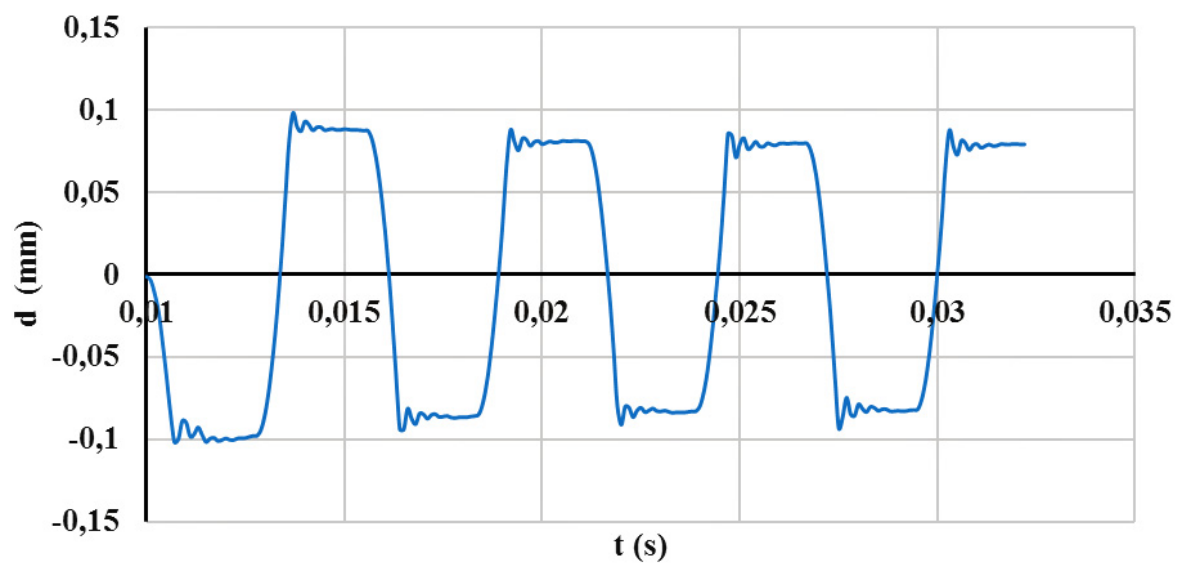

Fig. 8. Screw deflection $d$.

\subsection{Results of simulation by finite element method}

Figure 7 represents the sinusoidal displacement $u_{x}$ imposed at the end of the lower plate, from the beginning of step 3 of the simulation $(t=0.01 \mathrm{~s})$.

The screw deflection, i.e. the difference $d$ between displacements in the $x$ direction of the two ends of the screw, is shown in Figure 8. At the very beginning, both ends of the screw are driven by the plates, and the deflection follows the sinusoidal shape of the movement. When the bending is sufficient, at about $d=-0.1 \mathrm{~mm}$, the nut slides on the lower plate, and the deflection remains roughly constant. When the movement reverses, sliding ceases and the nut is driven again by the sinusoidal movement, toward the positive direction. Sliding resumes in the opposite direction at about $d=0.09 \mathrm{~mm}$, then the deflection remains roughly constant, and so on. The deflection necessary to initiate the sliding decreases slightly at each movement because of the progressive loosening of the assembly, which gradually reduces the tension of the screw and the normal force acting on the bearing faces.

Figure 9 shows the contact status at a characteristic point of the bearing surface of the nut on the lower plate. Because of the massive character of these parts, the contact status evolves rapidly on the surface and transition occurs almost instantaneously on the whole surface. This contact initially sticks, and we can see the sliding peri- ods corresponding to the periods of constant deflection of the screw in the previous figure. The sticking duration increases slowly, with the progressive loosening of the assembly.

Figure 10 represents the displacement of the nut relative to the lower plate, which is the sliding of the nut on its bearing. During the sliding periods, the displacement of the nut reproduces a part of the imposed sinusoidal movement.

When the nut slips both on the lower plate and on the screw, it is only guided by the forces coming from the thread and the bearing surface. Then the torque coming from the helix angle of the thread can deflect the movement, inducing a rotation of the nut and unscrewing the assembly, as previously indicated in Section 2.2.

Figure 11 shows the rotation of the nut, and Figure 12 shows the evolution of tensile stress in the axial direction, $z$, in the middle area of the bolt shank. These graphs are drawn from the very beginning of the simulation, showing the 3 steps already mentioned. In the first step, we can see an increase of the axial stress, corresponding to the tightening phase, without any rotation. The second step allows stabilization and shows no change in rotation or stress.

Soon after the beginning of the third step, the nut begins to slide, and it rotates simultaneously in the unscrewing direction. This is accompanied by a decrease of 


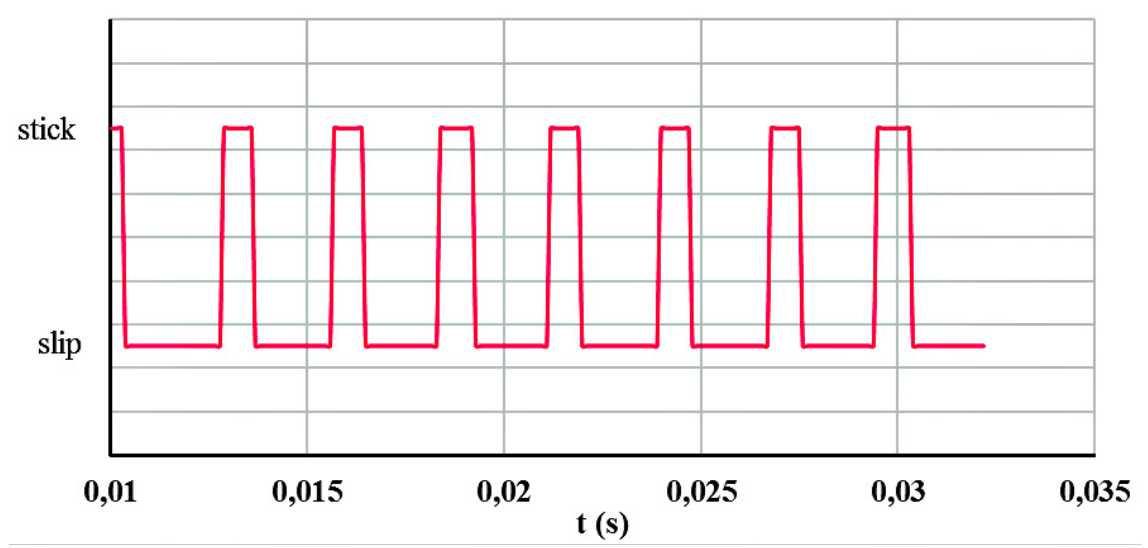

Fig. 9. Contact status of nut on lower plate.

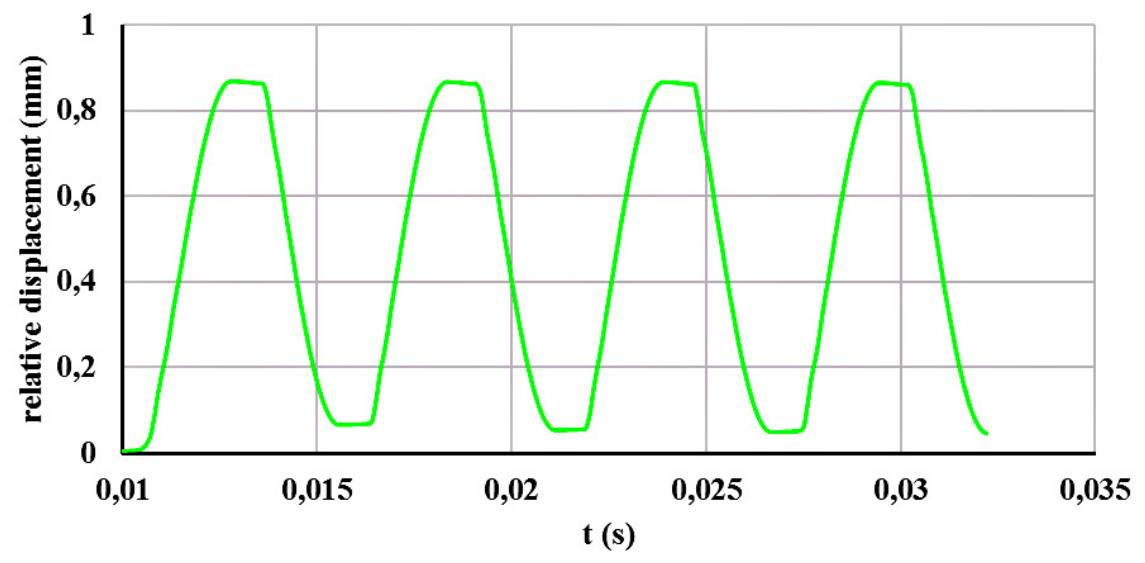

Fig. 10. Sliding of nut on its support.

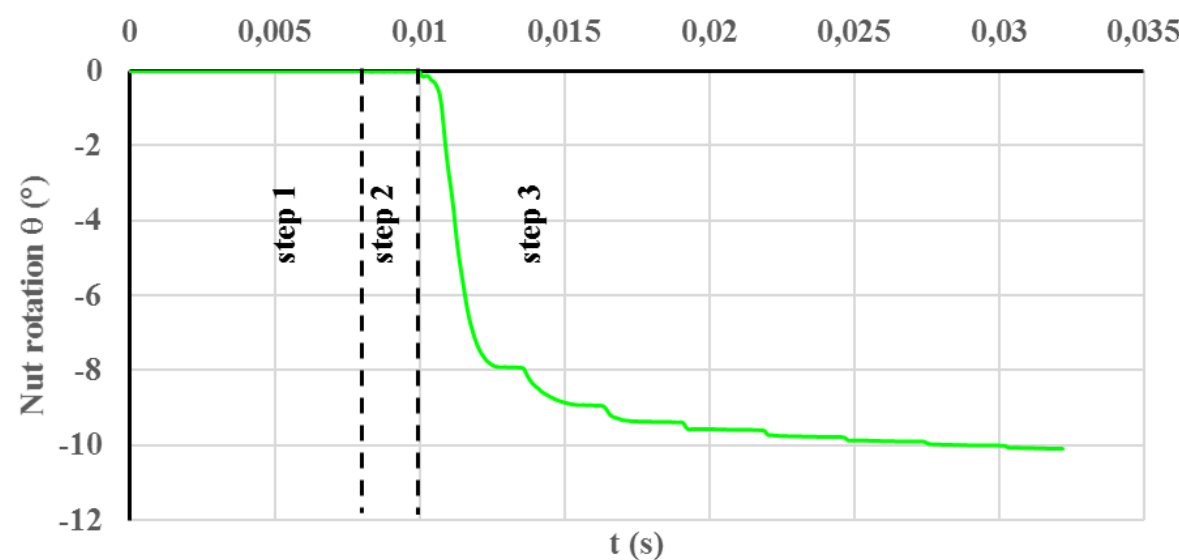

Fig. 11. Nut rotation $\theta$.

the axial stress, expressing the progressive loss of preload. At the end of the first slipping phase, at $t=0.013 \mathrm{~s}$, the rotation reaches $8^{\circ}$, and the axial stress has already lost about $36 \%$ of its initial value. The torque also decreases, so the following slipping phases generate less rotation and less loss of stress. Finally, after 4 sinusoidal cycles, unscrewing reaches $10^{\circ}$ and loss of load reaches $46 \%$. From there on, unscrewing and loosening of the assembly continue very slowly.

\section{Simulation of self-loosening by a 1D numerical simulation}

We now present a much simpler model, which describes the self-loosening phenomenon through a more global approach. This greatly reduces the computing time, the results are simpler and clearer, and the analysis is facilitated. 


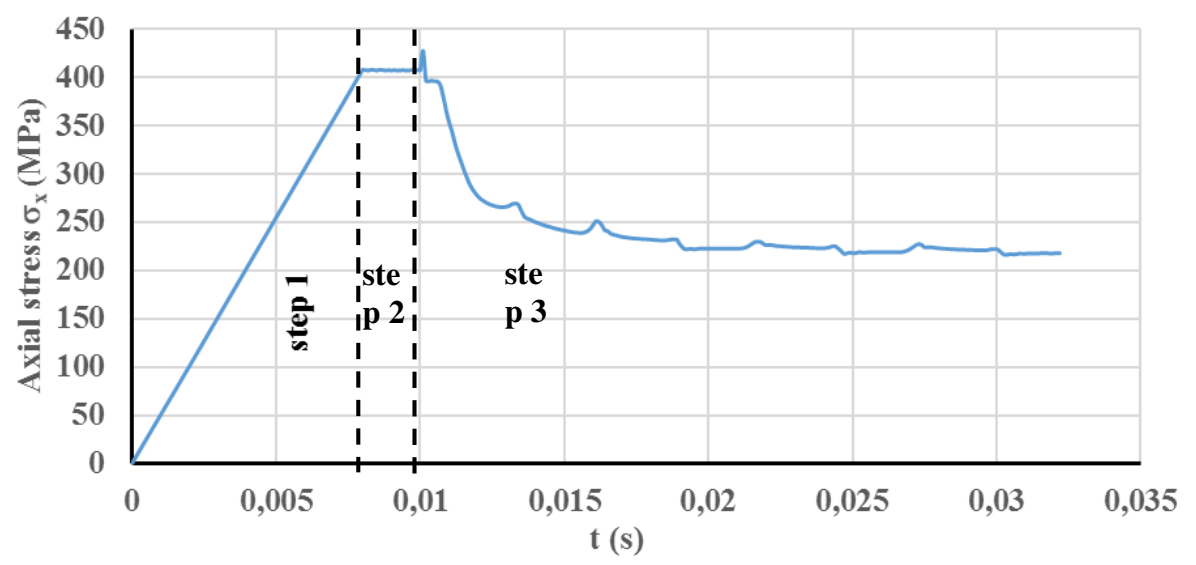

Fig. 12. Axial stress $\sigma_{x}$ in bolt shank.

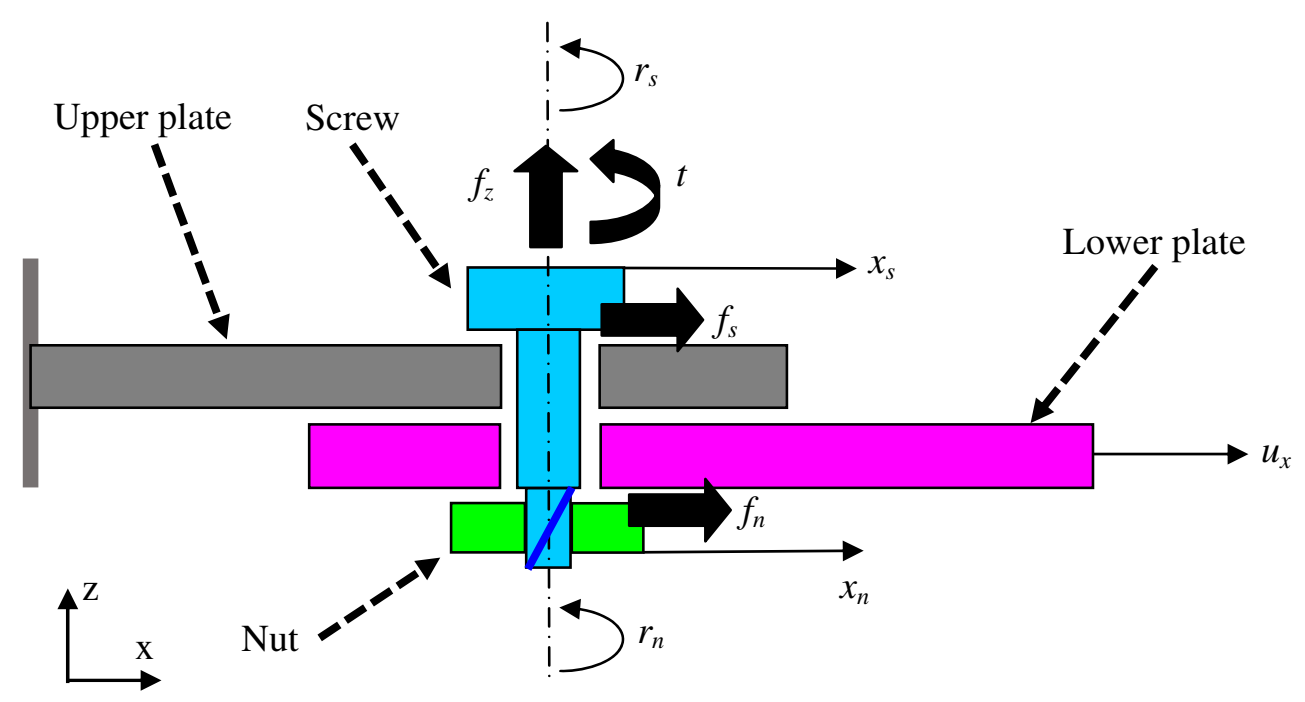

Fig. 13. Simple model.

\subsection{Simplified model with rigid bodies}

The simplified model used in this simulation is presented in Figure 13. It is made of 4 solid bodies: the upper and lower plates, the screw and the nut. The only recognized deformation is bending of the screw shank. There are only four degrees of freedom: translations $x_{\mathrm{s}}$ and $x_{\mathrm{n}}$, rotations $r_{\mathrm{s}}$ and $r_{\mathrm{n}}$, of the screw and the nut respectively. The lower plate moves by an imposed translational movement $u_{x}(t)$.

The axial load $f_{z}$ in the screw is initially equal to the preload $f_{z_{0}}$. It decreases linearly with the unscrewing angle $\left(r_{\mathrm{s}}-r_{\mathrm{n}}\right)$. Friction between the screw head and the upper plate generates a Coulomb friction force $f_{\mathrm{s}}$ on the screw, when friction between the nut and the lower plate generates a force $f_{\mathrm{n}}$ on the nut. The friction coefficient is greater under the screw head than under the nut so, in fact, there is no rotation of the screw and only 3 degrees of freedom effectively vary.

The helical connection between screw and nut is modelled by a simple link, without friction. Through the helix angle, the preload acting on the thread generates a torque about the $z$ axis, acting in opposite directions on the screw and the nut. The torque on the screw is $t$. It is proportional to the axial load $f_{z}$, and decreases during unscrewing.

When sliding appears between plates, the screw shank is subjected to a bending moment. We model its behaviour by a beam, clamped on plates at both ends, and we determine its deflection using the classical laws of strength of materials. We globally take account of the deformability of the screw head, the nut and the threads by increasing the length of this beam by a flat rate.

The screw and the nut have little mass so the inertial force generated is weak compared to the preload and friction forces. Their mass is therefore neglected and these parts are considered to be always in a state of static equilibrium.

\subsection{Cross slipping}

When slipping between any two parts occurs under the combined effect of two forces $f_{1}$ and $f_{2}$ acting in different directions, the movement is oriented toward the resultant force, and velocities $v_{1}$ and $v_{2}$ in each direction have the 

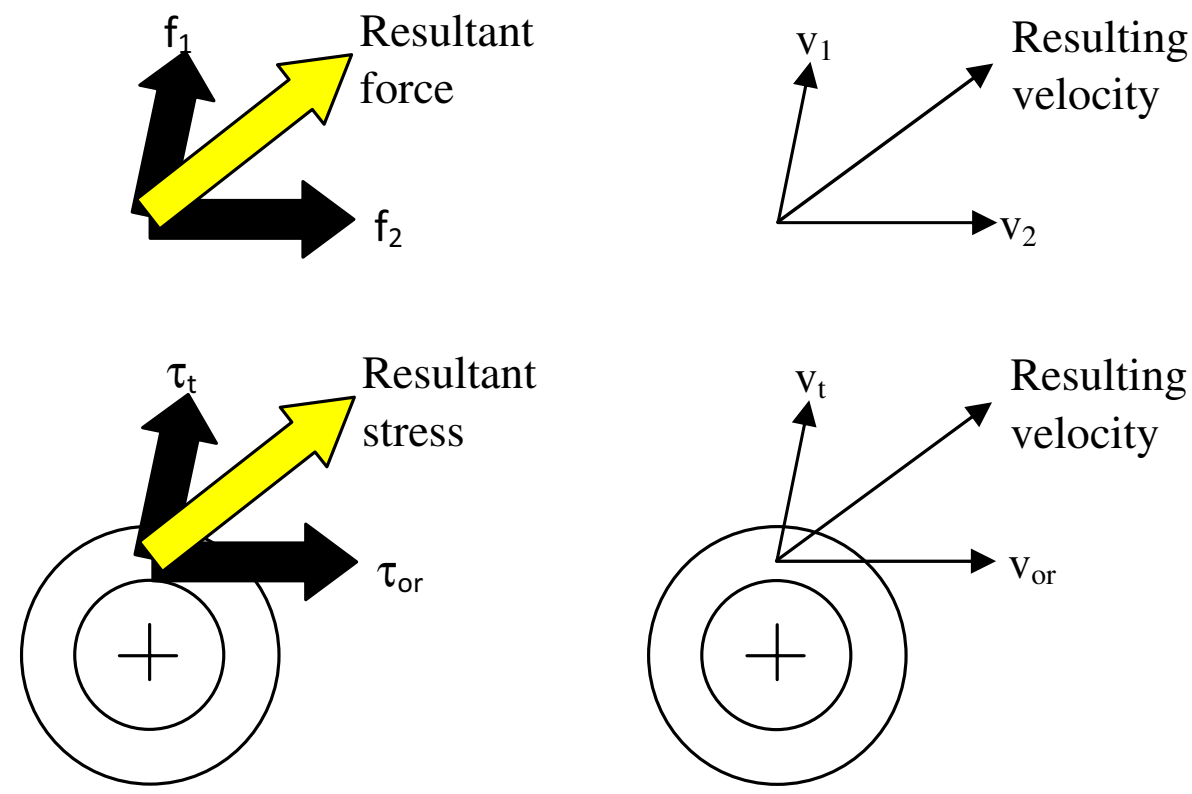

Fig. 14. Force, stress and velocity during cross slipping.

same relation as the forces (Eq. (2)), as shown in the first part of Figure 14.

$$
\frac{v_{2}}{v_{1}}=\frac{f_{2}}{f_{1}}
$$

Likewise, slipping can occur at the bearing face of the nut in a bolted assembly, under the combined effect of a transverse force $f_{\mathrm{t}}$, due to bending of the screw shank, and a torque $t$, due to the preload acting on the thread, as seen previously in Section 2.2 and Figure 3. The resulting slipping movement consists of a translation and a rotation as shown in the second part of Figure 14, which represents the annular bearing surface of the nut on the plate. The relation between the transverse velocity $v_{\mathrm{t}}$ and the orthoradial one $v_{\text {or }}$ is the same as that between transverse stress $\tau_{\mathrm{t}}$ due to $f_{\mathrm{t}}$, and orthoradial stress $\tau_{\text {or }}$ due to $t$, as in Equation (3).

$$
\frac{v_{\mathrm{t}}}{v_{\mathrm{or}}}=\frac{\tau_{\mathrm{t}}}{\tau_{\mathrm{or}}}
$$

For a sliding area $A$ with a mean radius $R$, and a rotational speed $\omega$, we have Equation (4):

$$
\frac{v_{\mathrm{t}}}{\omega R}=\frac{\frac{f_{\mathrm{t}}}{A}}{\frac{t}{R A}}=\frac{f_{\mathrm{t}}}{\frac{t}{R}} \quad \text { whence } \omega=\frac{v_{\mathrm{t}}}{f_{\mathrm{t}}} \frac{t}{R^{2}}
$$

If we consider that the orthoradial force due to torque remains small compared to that required to cause slipping, the sticking or sliding state is determined only by the transverse force $f_{t}$ acting in accordance with Coulomb's law of friction.

In the case of displacement of the nut on the lower plate:

- the rotational speed $\omega$ is the derivative of the rotation $r_{\mathrm{n}}$ of the nut;
- the transverse velocity $v_{\mathrm{t}}$ is the slipping velocity of the nut, it is the derivative of the relative displacement $\left(x_{\mathrm{n}}-u_{x}\right)$ between nut and plate;

- the transverse force $f_{\mathrm{t}}$ is the shear force $f_{\mathrm{n}}$ in the screw shank, which results from its bending;

- the torque $t$ comes from the thread.

We obtain Equation (5), which describes the rotational movement $r_{\mathrm{n}}$ of the nut.

$$
\frac{\mathrm{d} r_{\mathrm{n}}}{\mathrm{d} t}=\frac{\mathrm{d}\left(x_{\mathrm{n}}-u_{x}\right)}{\mathrm{d} t} \frac{1}{f_{\mathrm{n}}} \frac{t}{R^{2}}
$$

\subsection{Implementation of simulation}

The simulation was implemented using Matlab ${ }^{\circledR}$ with the classical function ode23 which performs step by step integration along an explicit Runge-Kutta $(2,3)$ pair of Bogacki and Shampine. It is adequate for solving moderately stiff problems. The flowchart in Figure 15 presents the successive operations that are included in the integration loop controlled by the ode23 function.

The movement imposed on the lower plate was the same as previously defined in Figure 6.

\subsection{Results of 1D simulation}

This simulation does not need preliminary steps. So the sinusoidal movement begins at $t=0 \mathrm{~s}$, and lasts for 4 periods until $t=0.023 \mathrm{~s}$. Figure 16 shows the results, with approximately the same disposition as the previous simulation using the finite element method.

The bending of the screw, the sequence of sticking and slipping states, and the slipping of the nut on its support are very similar to those of the previous simulation. 


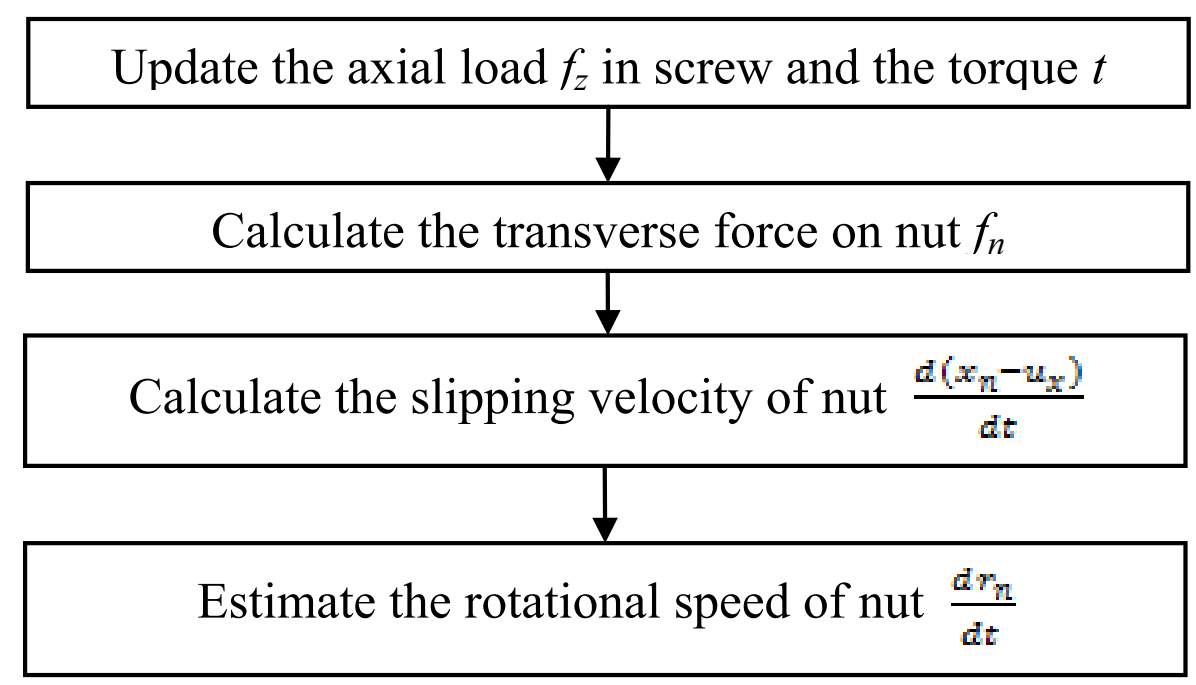

Fig. 15. Operations in the integration loop.

In both simulations, the screw head remains fixed relative to the upper plate, deformation of the screw remains weak, and most of the imposed displacement is absorbed by slipping under the nut. The main differences are:

- The screw deflection decreases faster in the 1D simulation. This may come from the different distribution of stiffness along the screw, the effect of which varies with the evolution of the axial load.

- Rotation of the nut, i.e. unscrewing, progresses in a different way: it slows down rapidly in the FEM simulation but it continues at a constant speed in the $1 \mathrm{D}$ simulation. This is due to the lack of friction in the thread for the 1D model. In this model, both the unscrewing and the resisting torques are proportional to the axial load on the bolt. So when this load decreases, the equilibrium remains unchanged and unscrewing continues in the same way. In the FEM model, friction in the threads comes from both the axial load and the bending of the screw. When the axial load decreases, friction in the threads remains high and this slows down unscrewing.

Finally, this model has some limitations:

- Deformations of the assembled parts are neglected;

- The transversal force on the screw and nut is taken to be much greater than the orthoradial force due to the torque coming from the helix angle of the thread. This assumption is correct for bolts with fine thread and wide bearing surfaces but it may become questionable for coarser thread and narrower bearing surfaces;

- Friction in the thread is neglected. This friction comes from both the bending and the tension of the screw, and it seems difficult to take it into account without a complicated, detailed model.

However, early symptoms of failure are similar between the $1 \mathrm{D}$ and $3 \mathrm{D}$ models. The simplified model is intended to be used to look for solutions in the context of preliminary design, by early detection of loss of preload and risk of loosening. In this application, it can be very useful.

\section{Conclusion}

In this paper, two methods have been used to simulate self-loosening of a bolted joint subjected to a transversal load coming from a displacement imposed between the assembled parts. First we used the finite element method to make a detailed model, taking the details of geometry and friction in all parts into account. We introduced the bolt preload by means of an angular rotation applied to the screw head. Calculation time was long, and results were obtained where unscrewing slowed down when the load in the screw decreased.

Then a $1 \mathrm{D}$ simulation method was used, where the geometry and the phenomena were greatly simplified. Bending of the screw shank was the only deformation taken into account, and no friction was considered in the thread. This led to only 4 degrees of freedom and calculation was almost instantaneous. The results were very near the previous ones, with the important difference that unscrewing progressed at roughly constant speed although the bolt load decreased. This was because the description of the threaded joint was too simple and did not take account of any friction on the thread flank. It seems difficult to consider thread flank friction without a much more complicated and detailed model. This shortcoming in the 1D method prevented the course of unscrewing from being predicted but the method nevertheless answered the main practical question: will the assembly unscrew or not?

The closeness of the results obtained through the two models is encouraging. The next step will be to compare their behaviour with an experimental approach, in order to validate and fit the models.

We expect that our FEM model will be useful to explore the loosening of some critical systems, where a long calculation time is acceptable to obtain precise results. We also hope that our simplified model will serve to check the effect of multiple parameters on loosening, via a parametric study with approximate results but very rapid calculation. 
O. Ksentini et al.: Mechanics \& Industry 17, 507 (2016)

Displacement ux at the end of the lower plate

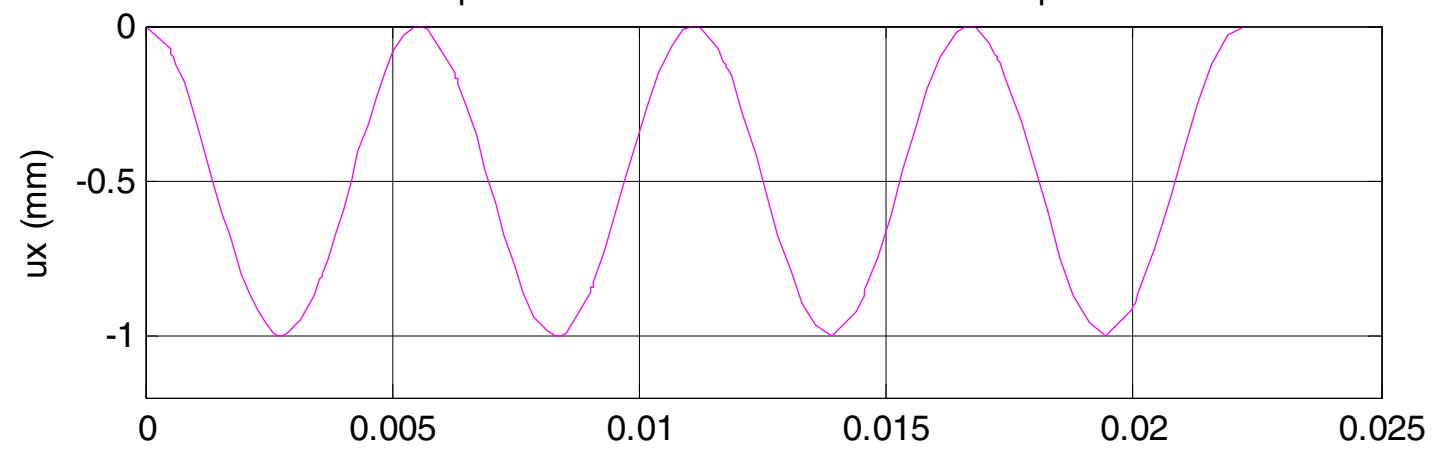

$\mathrm{t}(\mathrm{s})$

Screw deflection d
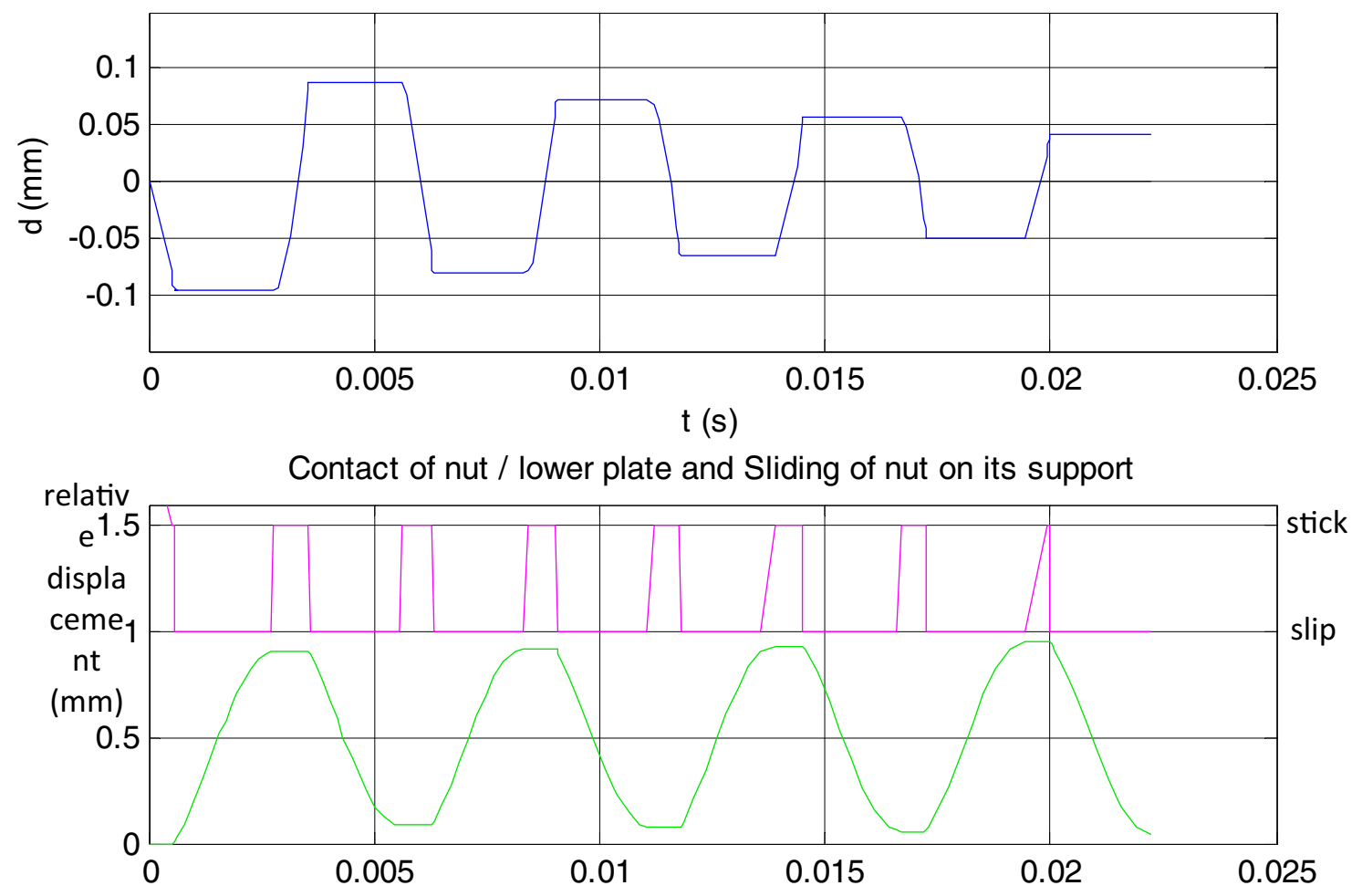

$\mathrm{t}(\mathrm{s})$

Nut rotation $\theta$

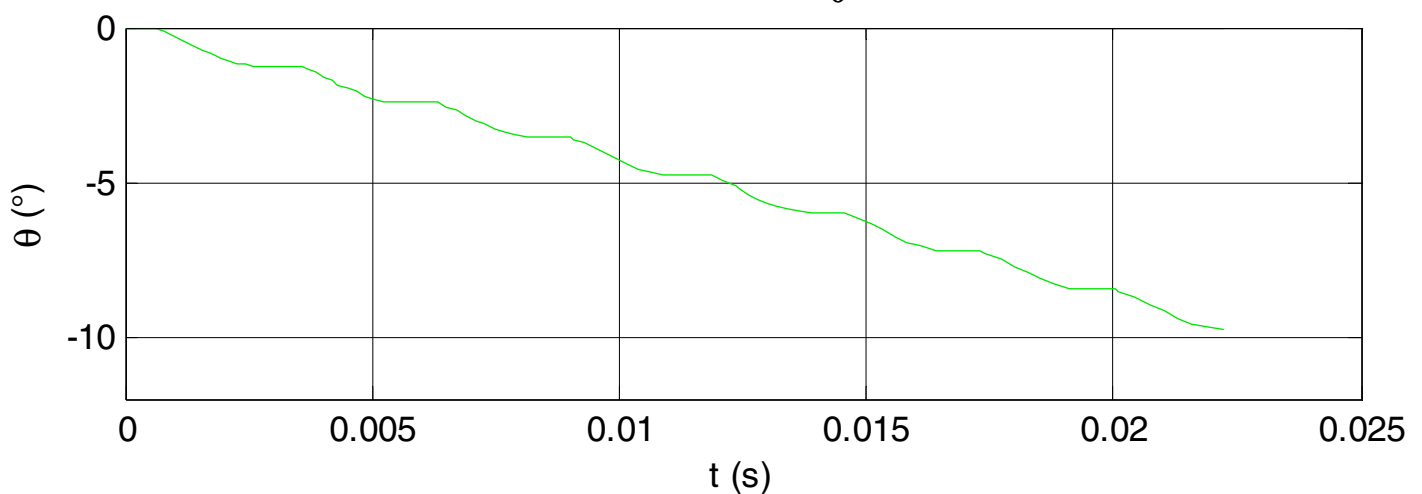

Fig. 16. Results of 1D simulation. 


\section{References}

[1] J. Guillot, Calcul des assemblages vissés : Assemblages de pièces planes de faibles épaisseurs. Partie 1, Techniques de l'Ingénieur, BM5564, 2010, pp. 1-20

[2] J. Guillot, Calcul des assemblages vissés : Assemblages de pièces planes de faibles épaisseurs. Partie 2, Techniques de l'Ingénieur, BM5565, 2011, pp. 1-16

[3] D.P. Hess, S.V. Sudhirkashyap, Dynamic loosening and tightening of a single bolt assembly, J. Vib. Acoust. 119 (1997) 311-316

[4] D. P. Hess, Vibration and shock induced loosening, In the book: J. H. Bickford and S. Nassar, Handbook of bolts and bolted joints, Marcel Dekker, 1998

[5] H. Aziz, Étude du dévissage spontané des assemblages boulonnés, Ph.D. thesis, Institut National des Sciences Appliquées de Toulouse, 2003

[6] G.H. Junker, New criteria for self-loosening of fasteners under vibration, Society of Automotive Engineers, New york, 1969

[7] A. Bhattacharya, A. Sen, S. DAS, An investigation on the anti-loosening characteristics of threaded fasteners under vibratory conditions, Mech. Mach. Theory 45 (2010) $1215-1225$
[8] X. Jiang, Y. Zhu, J. Hong, X. Chen, Y. Zhang, Investigation into the loosening mechanism of bolt in curvic coupling subjected to transverse loading, Eng. Failure Anal. 32 (2013) 360-373

[9] M. Zhang, Y. Jiang, C. H. Lee, Finite Element Modeling of Self Loosening of Bolted Joints, Trans. ASME 129 (2007) 218-226

[10] G. Dinger, C. Friedrich, Avoiding self-loosening failure of bolted joints with numerical assessment of local contact state, Eng. Failure Anal. 18 (2011) 2188-2200

[11] S.A. Nassar, B.A. Housari, Effect of Thread Pitch and Initial Tension on the Self-Loosening of Threaded Fasteners, Trans. ASME 128 (2006) 590-598

[12] S.A. Nassar, X. Yang, A Mathematical Model for Vibration-Induced Loosening of Preloaded Threaded Fasteners, J. Vib. Acoust. 131 (2009) 1-13

[13] X. Yang, S.A. Nassar, Z. Wu, Criterion for Preventing Self-Loosening of Preloaded Cap Screws Under Transverse Cyclic Excitation, J. Vib. Acoust. 133 (2011) 1-11

[14] Dassault Systèmes - Simulia, ABAQUS Software version 6.13 User's Manual, 2013 